\title{
Human germline gene editing: Recommendations of ESHG and ESHRE
}

\author{
Guido de Wert ${ }^{1}$ - Guido Pennings ${ }^{2}$ - Angus Clarke $\mathbb{D}^{3}$ - Ursula Eichenlaub-Ritter ${ }^{4}$ - Carla G. van $\mathrm{El}^{5}$. \\ Francesca Forzano $^{6}$ - Mariëtte Goddijn ${ }^{7}$ - Björn Heindryckx ${ }^{8}$. Heidi C. Howard ${ }^{9}$ - Dragica Radojkovic ${ }^{10}$. \\ Emmanuelle Rial-Sebbag ${ }^{11}$. Basil C. Tarlatzis ${ }^{12} \cdot$ Martina C. Cornel $\mathbb{1}^{5}$ On behalf of the European Society of \\ Human Genetics and the European Society of Human Reproduction and Embryology
}

Received: 23 August 2017 / Accepted: 5 September 2017 / Published online: 12 January 2018

(c) European Society of Human Genetics 2018

\begin{abstract}
Technological developments in gene editing raise high expectations for clinical applications, first of all for somatic gene editing but in theory also for germline gene editing (GLGE). GLGE is currently not allowed in many countries. This makes clinical applications in these countries impossible now, even if GLGE would become safe and effective. What were the arguments behind this legislation, and are they still convincing? If a technique can help to avoid serious genetic disorders, in a safe and effective way, would this be a reason to reconsider earlier standpoints? The European Society of Human Reproduction and Embryology (ESHRE) and the European Society of Human Genetics (ESHG) together developed a Background document and Recommendations to inform and stimulate ongoing societal debates. After consulting its membership and experts, this final version of the Recommendations was endorsed by the Executive Committee and the Board of the respective Societies in May 2017. Taking account of ethical arguments, we argue that both basic and preclinical research regarding GLGE can be justified, with conditions. Furthermore, while clinical GLGE would be totally premature, it might become a responsible intervention in the future, but only after adequate pre-clinical research. Safety of the child and future generations is a major concern. Future discussions must also address priorities among reproductive and potential non-reproductive alternatives, such as PGD and somatic editing, if that would be safe and successful. The prohibition of human germline modification, however, needs renewed discussion among relevant stakeholders, including the general public and legislators.
\end{abstract}

Guido de Wert

g.dewert@maastrichtuniversity.nl

1 Department of Health, Ethics and Society, Research Institutes GROW and CAPHRI, Fac. of Health, Medicine and the Life Sciences, Maastricht University, Maastricht, The Netherlands

2 Bioethics Institute Ghent, Department of Philosophy and Moral Science, Ghent University, Ghent, Belgium

3 School of Medicine, Cardiff University, Cardiff, UK

4 Institute of Gene Technology/Microbiology, Faculty of Biology, University of Bielefeld, Bielefeld, Germany

5 Department of Clinical Genetics, Section Community Genetics, and Amsterdam Public Health research institute, VU University Medical Center, Amsterdam, The Netherlands

6 Clinical Genetics Department, Guy's Hospital, Guy's and St Thomas' NHS Foundation Trust, London, UK
7 Center for Reproductive Medicine, Department of Obstetrics and Gynecology, Academic Medical Center, AmsterdamZuidoost, The Netherlands

8 Ghent-Fertility and Stem cell Team (G-FaST), Department for Reproductive Medicine, Ghent University Hospital, Ghent, Belgium

9 Centre for Research Ethics and Bioethics, Uppsala University, Uppsala, Sweden

10 Laboratory for Molecular Biology, Institute of Molecular Genetics and Genetic Engineering, University of Belgrade, Belgrade, Serbia

11 University Paul Sabatier Toulouse, Toulouse, France

12 1st Department of Obstetrics \& Gynecology, School of Medicine, Aristotle University of Thessaloniki, Thessaloniki, Greece 


\section{Introduction}

Recent research and expected further studies in gene editing raise high expectations, especially regarding possible therapeutic applications in humans. Most promising is the prospect of somatic gene editing, which may prove to be a game changer not only in the treatment of a whole range of serious hereditary disorders, especially Mendelian ones, but also in the treatment of cancer and infectious diseases. Over 5000 Mendelian diseases are identified whereas currently treatment is available for only a small minority of these. At the same time, the possibility of a future application in the human germline raises serious concerns. In previous decades, legislation has been adopted that does not allow changes to the human germline. What were the arguments behind this legislation, do they still apply and are they still convincing? If a technique can help to avoid serious genetic disorders (with severe effects on quality of life or life span) in a safe and effective way, would this be a reason to reconsider earlier standpoints? Discussion with relevant stakeholders is needed, including professional health care workers, patients and different groups of lay public, plus legal, and ethical experts. Recently, initiatives have been taken worldwide to exchange views and re-ignite the debate about responsible governance and approaches to innovation using human gene editing. The European Society of Human Genetics (ESHG) and the European Society of Human Reproduction and Embryology (ESHRE) consider it to be their professional responsibility to contribute to further discussion by means of a set of Recommendations, based on a Background document, focusing on human germline gene editing (GLGE).

The aim of this contribution is to inform and stimulate ongoing societal debates, as well as provide guidance, taking into account: the technical aspects of GLGE, its different possible applications, relevant clinical experience regarding the handling of reproductive risk, legal regulations, and the ethical and societal issues and concerns linked with GLGE. Because of the importance of the latter, both ESHG and ESHRE invited their relevant committees (respectively, the Public and Professional Policy Committee of ESHG and the Ethics Committee of ESHRE) to take the lead in writing the Background document and Recommendations. Drafts were prepared by a joint writing group and were discussed in both committees and in a joint meeting of the two societies on September 20, 2016. Next, a draft of the Recommendations was posted online from October 17 until December 2, 2016 and was presented at the ASHG meeting in Vancouver. The Background document and the Recommendations have been posted online to solicit comments from the membership of both ESHG and ESHRE from April 3 until May 8, 2017. The authors integrated the suggestions where appropriate and the
Recommendations were endorsed by the Board of ESHG and the Executive Committee of ESHRE. This Document is of a provisional nature, and is to be re-evaluated regularly, taking account of relevant scientific developments, possible future clinical experiences, and evolving discussions in society as a whole as well as ethical reflection. The Recommendations should be understood against the argumentation provided in the Background document $[1,2]$. We strongly recommend also reading this Background document and will refer to relevant sections below.

\section{Recommendations}

In preparing this Document, it was considered crucial to make a distinction between non-reproductive GLGE in basic research, non-reproductive GLGE in pre-clinical research and possible future clinical (reproductive) GLGE.

\section{Non-reproductive GLGE in basic research}

Non-reproductive GLGE primarily concerns the context of basic research. Although a sharp demarcation between basic research and pre-clinical research is difficult to make, basic research in this context is characterized by a focus on fundamental questions regarding human embryology and the methods applied in gene editing. Reproductive treatments in health care and adequate patient counseling may be served by a better knowledge of early embryo development. There are good reasons to allow basic research in this area, subject of course to societal oversight and taking account of relevant ethical guidelines and (inter-)national legal regulations.

The research use of human somatic cells and (precursor cells of) gametes is less controversial than human embryo research in vitro. The Oviedo Convention categorically prohibits the making of human embryos specifically for research purposes ('research embryos'). Several European countries rely on the use of spare embryos left over after IVF procedures only. If necessary and possible, the use of spare embryos over embryos created for research should be the preferred option. However, also from an ethical point of view, a prohibition to make such embryos may be contested. As elaborated in the Background document, the making of research embryos could be morally justified, subject to ethical, legal and societal oversight, if the research question cannot be adequately addressed on the basis of spare embryos only and if research embryos are necessary to reach the aim(s) of scientifically sound and robust research. Given the sensitivity of human germline interventions, the specific consent of the providers of the gametes and embryos to such basic research use should be obtained. 


\section{Non-reproductive germ-line gene editing in preclinical research}

Only after acquiring robust knowledge from basic research, might future clinical applications be considered, which would require further societal and professional discussions. Both for scientific and moral reasons, as a precondition for any potential clinical applications of GLGE, adequate preclinical research on GLGE is necessary. Preclinical research, involving both animal and human embryo research, is an important element of the moral framework for the introduction of new, experimental, reproductive technologies generally. Given the specific sensitivity of GLGE, such research would have to take place under ongoing monitoring and societal oversight. Pre-clinical GLGE research would involve investigation of the safety (e.g. possible off-target effects or epigenetic effects) and effectiveness of gene editing in view of possible future reproductive applications of GLGE in gametes, zygotes or preimplantation embryos. Such research is important in order to identify and eliminate, or at least reduce, avoidable risks for any future children thus conceived.

Conceptually, the term pre-clinical research alludes to potential clinical applications at least being considered, if not intended. However, pre-clinical research is a necessary, but not sufficient prerequisite for future applications, and such applications do certainly not automatically follow from allowing pre-clinical research as is outlined in the Background document.

'Comprehensive' genetic testing of embryos (preimplantation genetic testing, PGT) using whole genome sequencing (WGS) might be an integral part of adequate pre-clinical research on the safety and specificity of GLGE to investigate potential off-target effects. The issue of how to handle possible incidental findings regarding the genetic make-up of the providers of the gametes or embryos should be addressed in the informed consent process, taking account of relevant guidelines.

According to the Clinical Trials Regulation EU No.536/2014, Article 90, modifications to the germ line are not allowed. It must be clarified what this implies for preclinical studies.

\section{Reproductive GLGE}

Potentially in the future, depending on the outcomes of basic and pre-clinical research and taking account of societal views, risks and implications (see below), moving the technique towards the clinic may be considered. If so, this should be embedded in a formal research trajectory. According to the Clinical Trials Regulation EU No.536/2014, Article 90 'No gene therapy clinical trials may be carried out which result in modifications to the subject's germ line genetic identity.' The implication of this regulation, is that adequate clinical GLGE research is impossible in the European Union (EU). Potentially this would also apply to preclinical research. Meanwhile, the clinical applications may take place outside the EU and in some cases may be carried out without proper research protocols and oversight. Given the technological development and the ethical analysis as described in the background document comparing GLGE to other available reproductive options, the time has come to discuss the rationale and consequences of the Clinical Trials Regulation.

If shown to be safe and effective, clinical GLGE may come to have important benefits for prospective parents at high risk of having a child affected by a serious genetic disorder and for whom, for example, PGD is not a real option.

Categorical deontological objections to clinical GLGEin terms of being at odds with, for example, naturalness, human dignity, or the preservation of the human gene pool as a common heritage - are often used both in public debate and legal discourse. While these objections may be relevant for possible (mostly rather theoretical) enhancement-like applications of GLGE, they seem unconvincing when it comes to possible applications of GLGE with a clear therapeutic or preventive aim, as elaborated in the Background document. A better understanding of these objections, and the context in which they are used, is needed to inform future policy decisions, public debate and the counseling of individual patients.

Consequentialist objections to reproductive GLGE, regarding both health risks (3.1) and societal concerns (3.2), merit more scrutiny and debate.

\section{Health risks}

In the context of GLGE health risks should be taken to refer to not only those affecting the first generation but also possible subsequent ones. Different types of possible adverse effects (off-target and pleiotropic, genetic, and epigenetic) need investigation. In view of the many unknowns, any use of GLGE methods for clinical purposes, including any reproductive use of gametes derived from edited pluripotent somatic cells, should be regarded as premature and therefore at present unacceptable.

Clinical applications can only become morally justified if adequate pre-clinical safety research, including (human) embryo research, shows clinical GLGE to be sufficiently safe and efficient. The proper standard for the evaluation of possible residual risks ('how safe is safe enough in order to start clinical applications?') needs specific assessment after further debate and clarification.

If comprehensive PGT of edited embryos on the basis of WGS would be included as a safeguard in future clinical 
GLGE, this testing should be focused on possible off-target effects, taking into account potential mosaicism. A possible broadening of the interpretation of the raw data generated by such PGT raises complex additional ethical issues and would need further multidisciplinary analysis and debate. The proportionality of such broader analysis should not be taken for granted, and it may generate more questions than answers, as elaborated in the Background document.

Furthermore, any potential future reproductive GLGE would require prospective data collection of reproductive outcomes and long-term follow-up studies on the health of children thus conceived. Possible practical barriers and limits (in terms of, for example, lack of funding or tensions between long-term follow-up and familial and children's privacy) may render this challenging, as with long-term follow-up of children conceived through new reproductive technologies generally.

\section{Societal concerns}

The major societal risks often mentioned in this context are inequity, the undermining of reproductive autonomy, the position of people affected with impairments or disabilities, and possible misuse of GLGE for non-medical applications.

The disability rights critique forcefully reminds society of its responsibilities towards people with disabilities, more particularly its obligation to remove barriers for inclusion, but it should not be used as an argument against the development of medical therapies, including gene editing, irrespective of whether it concerns somatic gene editing or GLGE.

Equal access to health care has to be decided at the level of society as a whole. Public funding, as some countries have provided for PGD, can mitigate the concerns regarding inequity. If limited funding is available for health care, prioritization is needed. It is conceivable that somatic gene editing will be prioritized over GLGE as many serious health problems could be targeted and it might be a proportionate (albeit temporary) approach to treatment. The definition and specification of seriousness may be a challenge and needs to be assessed within specific national ethical rules and regulations.

Reproductive autonomy should be maintained and respected by both adequate counseling and provisions for disabled people. Moreover, while some fear the undermining of reproductive autonomy, it should be noted that GLGE may well promote the reproductive autonomy of prospective parents at high risk of having a child affected with a serious disorder, as it would increase the number of reproductive options (see below).

In order to ensure strong societal and professional oversight, the experience with regulating PGD and other reproductive technologies should help build a sound strategy for regulating possible future clinical applications of GLGE, including a licensing system for clinics involved, quality controls and obligatory regular reporting by licensed clinics of their handling of requests for GLGE. If clinical GLGE does come to be considered sound, priority should be given to the editing of highly penetrant genes causing serious disorders. As the distinction between serious and less serious disorders is unclear, feeding fears of a slippery slope, further multidisciplinary reflection on the demarcation of serious disorders is needed. In addition, the distinction between therapy and enhancement is not always clear-cut and decisions will need to be made about intermediate subtypes of medical enhancement, such as strengthening the human immune system or editing carrier status for recessive disorders or structural aberrations. With regard to fears about possible future 'designer babies', it is important to acknowledge that the prospect of enhancing complex traits (such as intelligence) is to a large extent science fiction, and that possible efforts to enhance traits would run a disproportional risk of antagonistic (harmful) pleiotropic effects. Public debate and education is needed to lower the risk of commercial companies seeking to exploit prospective parents' (unrealistic) preference for a 'perfect child'.

In view of the medical and societal risks of, and concerns regarding, GLGE it is important to take account of other reproductive options for people at high risk of having an affected child. Considering the preference of most prospective parents to have a healthy child who is genetically related to both of them, PGD aimed at the selective transfer of an unaffected embryo may be a good 'preventive' option in most cases. Still, there may be situations where GLGE might be justified, depending upon the genetic disorder under consideration, the prospective parents' genetic makeup, their experiences with clinical PGD, their weighing of the possible risks and burdens of a further cycle of IVF/PGD, and their moral and religious preferences, including their possible wish to minimize embryo loss. A further ethical and societal evaluation of relevant aspects, including the possible health risks of GLGE, is needed to define the potential future indications for clinical GLGE as an alternative to PGD that aims to selectively transfer an unaffected embryo.

Possible future routine comprehensive PGT of IVFembryos using WGS, aimed at selecting 'the best embryo' for transfer, needs proactive scientific, ethical, and societal debate. Such testing could well, assuming a further improvement of the efficiency of editing (post-zygotic) embryos, function as a driver for future routine GLGE, at least among some (wealthy) social groups. After all, there will always be potentially pathogenic variants, as all embryos, like humans, are 'fellow mutants'. While this scenario would be problematic in view of the pleiotropic 
risks of GLGE, it does at the same time urge society even more strongly to engage in a more principled debate about the ethics of, and policymaking regarding, the conceptually and morally grey area between therapeutic, preventive, and enhancement GLGE.

\section{Governance}

A process of ongoing public debate about material and procedural ethical and societal issues raised by both nonreproductive and reproductive human GLGE is of the utmost importance. Such debate should be based on sound scientific evidence as well as sound ethical, legal, and social reflection in such a manner that many different stakeholders can understand and take part. A strategic plan, including the funding, and practical and temporal aspects should be devised to ensure that such debates are prioritized and undertaken at the same time that the science and policy discussions evolve. Multi-stakeholder debates should be inclusive; apart from scientists and clinicians, other stakeholders should be invited to participate, including patients' organizations, different lay groups of the public, policymakers, and scholars in the medical humanities.

These current Recommendations build a first, joint, contribution of both ESHRE and ESHG to the suggested ongoing trajectory of professional and public deliberations. The Recommendations are of a provisional nature and are to be evaluated regularly and systematically to facilitate flexibility in approaches and regulation.

\section{Summary}

What does this study mean for patients?

- This paper contains Recommendations from ESHRE and ESHG concerning human gene editing of sperm, oocytes, and embryos.

- The Societies recommend that there are good reasons for more research into this area, as this approach has potential for helping people with hereditary diseases. However, it would make permanent changes to the genetic make up of edited offspring, so any research into this method of gene editing must be carefully monitored and overseen. Only after such research had ensured the safety and efficacy of the method would it even be considered for clinical use to help people with hereditary diseases. There would need to be provision for followup studies and further research, as well as proper public debate.

- The details of the initial Recommendations from the Societies are contained in this paper, but they make it clear that these are likely to need re-evaluation in the future.

Acknowledgements The authors thank the persons who commented on earlier versions of these Recommendations or the Background document to these Recommendations, especially the members of the Public and Professional Policy Committee of ESHG and Ethics Committee of ESHRE and Erika Kleiderman, Bartha Maria Knoppers, Raul Piña-Aguilar, Alessandra Renieri, Gijs Santen, and Kirmo Wartiovaara

Funding No external funding was obtained. Costs for the meetings were covered by ESHRE and ESHG.

\section{Compliance with ethical standards}

Conflict of interest The authors declare that they have no conflict of interest.

\section{References}

1. De Wert G, Heindryckx B, Guido Pennings G, et al. Responsible Innovation in Human Germline Gene Editing. Background document to the Recommendations of the ESHG and ESHRE. Eur $J$ Hum Genet. 2017.

2. De Wert G, Heindryckx B, Guido Pennings G, et al. Responsible Innovation in Human Germline Gene Editing. Background document to the Recommendations of the ESHG and ESHRE. Hum Reprod Open. 2017. 